\title{
Cyclin D1 expression in Bowen's disease and cutaneous squamous cell carcinoma
}

\author{
YANYUN SHEN, JINHUA XU, JIN JIN, HUI TANG and JUN LIANG \\ Department of Dermatology, Huashan Hospital, Fudan University, Shanghai 200040, P.R. China
}

Received December 6, 2013; Accepted January 1, 2014

DOI: $10.3892 / \mathrm{mco} .2014 .273$

\begin{abstract}
Cyclin D1 is a member of the G1 cyclin family that regulates the transition through the G1 phase of the cell cycle and is involved in the neoplastic transformation of certain tumors. This study was designed to investigate the expression of cyclin D1 in Bowen's disease (BD) and cutaneous squamous cell carcinoma (SCC). Biopsies of 30 cases with BD and 24 cases with SCC confirmed by histopathology were obtained from the Department of Dermatology of Huashan Hospital, Shanghai, China. EnVision immunohistochemical technology with a semiquantitative immunohistochemical score was applied to detect the expression of cyclin D1. Of the 24 specimens with SCC, cyclin D1 was found to be positive in $17(70.8 \%)$, whereas of the 30 specimens with BD, cyclin D1 was found to be positive in $13(43.3 \%)$. The expression of cyclin D1 was significantly higher in the SCC compared to that in the BD group. We did not observe a significant association of cyclin D1 expression with different pathological grades of SCC. In conclusion, cyclin D1 plays a significant role as a diagnostic marker in skin tumors and its overexpression was not found to be correlated with the degree of differentiation of SCC.
\end{abstract}

\section{Introduction}

The process of skin carcinogenesis involves a series of transitional events, ranging from hyperplasia, dysplasia and papilloma to invasive squamous cell carcinoma (SCC) (1). Gene products that are significantly associated with cell proliferation and cell cycle control may be interesting from a scientific viewpoint, since these genes and their protein products may represent targets for specific therapeutic approaches and may also be used as prognostic markers (2). One such protein is cyclin D1, which is a $45-\mathrm{kD}$ a protein encoded by the cyclin D1 (CCND1) gene on chromosome 11q13 (3). Cyclins play a key

Correspondence to: Dr Jun Liang, Department of Dermatology, Huashan Hospital, Fudan University, 12 Central Urumqi Road, Shanghai 200040, P.R. China

E-mail: liangjun1976@medmail.com.cn

Key words: cyclin D1, cutaneous squamous cell carcinoma, Bowen's disease, immunohistochemistry role in cell cycle control and are themselves regulated by cyclin-dependent kinases. The activation of cyclins leads to the activation of the cell cycle from the G1 to the S phase and consequently promotes cell proliferation. The overexpression of cyclin D1 leads to shortening of the G1 phase and increases independence from growth factors and abnormal cell proliferation, which may foster the occurrence of additional genetic alterations $(4,5)$. This induction of proliferation by cyclin D1, which is due to gene amplification, was previously identified in different types of human malignant tumors, including cancer of the breast (6), esophagus (7), liver (8), head and neck (3), lung (9), larynx (10), bladder (11), uterus (12), lymphoid tissue (13), melanoma $(14,15)$ and oral SCC $(4,16)$. Cyclin D1 has been considered to be an oncogene and it is hypothesized that it plays a significant role in the neoplastic transformation of certain tumors (14).

Skin cancer has recently become one of the most common human malignancies. However, the number of currently available studies on the role of cyclin D1 in human skin cancer, particularly in the process of carcinogenesis, is limited. The purpose of this study was to determine the expression of cyclin D1 in Bowen's disease (BD) and cutaneous SCC and assess whether there are any differences in the expression of these proteins in these two types of cancer. Elucidating the specific molecular and functional changes that underlie the development of these tumors may aid in designing specific treatment strategies and improve our understanding of the normal molecular pathways involved in skin development and function (17).

\section{Materials and methods}

Patients and tissue samples. Biopsy samples from a total of 54 patients were collected from Huashan Hospital over the last 3 years (Table I). All the diagnoses were confirmed by a dermatopathologist. A total of $30 \mathrm{BD}$ and 24 cutaneous SCC patients were enrolled. We also included 8 normal skin specimens to serve as controls and a bladder cancer sample as a positive control for cyclin D1.

This study was approved by the Ethics Committee of Huashan Hospital, Fudan University, Shanghai, China.

Immunohistochemistry. Immunostaining was performed on $3-\mu \mathrm{m}$ paraffin-embedded tissue sections. After the slides were deparaffinized in xylene and rehydrated, the endogenous 
Table I. Clinical data of the study.

\begin{tabular}{lcc}
\hline Variables & $\begin{array}{c}\text { Bowen's } \\
\text { disease }\end{array}$ & $\begin{array}{c}\text { Squamous cell } \\
\text { carcinoma }\end{array}$ \\
\hline Age (years) & $32-87$ & $33-91$ \\
Range & 65.4 & 66.2 \\
Mean & & \\
Gender & 11 & 16 \\
Male & 19 & 8 \\
Female & 30 & 24 \\
No. of biopsies & & 10 \\
Site of biopsy \\
(no. of specimens) & & - \\
Genitalia & 6 & 5 \\
Chest & 3 & 2 \\
Lower limbs & 8 & - \\
Back & 7 & $1-360$ \\
Buttocks & 1 & 67.8 \\
Upper limbs & 1 & 1 \\
Head and face & 2 & \\
Anal region & 1 & \\
Hands & 1 & \\
Approximate duration & & \\
of lesions (months) & & \\
Range \\
Mean
\end{tabular}

peroxidase was blocked with $3 \%$ hydrogen peroxide in methanol for $15 \mathrm{~min}$ and then washed 3 times with phosphate-buffered saline [phosphate-buffered saline (PBS; Bogoo Biotechnology Company, Shanghai, China), pH 7.4] for 5 min. The sections were incubated with rabbit anti-cyclin D1 monoclonal antibody (1:300 dilution; Epitomics, Inc., Burlingame, CA, USA) for $30 \mathrm{~min}$ at room temperature, then incubated at $4^{\circ} \mathrm{C}$ for $24 \mathrm{~h}$. After washing with PBS, the sections were incubated for $30 \mathrm{~min}$ with a horseradish peroxidase-labelled anti-rabbit secondary antibody (Dako, Glosrup, Denmark). Positive and negative immunohistochemistry controls were included. 3,3'-Diaminobenzidine (Vector Laboratories, Burlingame, CA, USA) was used for $10 \mathrm{~min}$ to visualize the immunolabelling. After washing, the sections were counterstained with haematoxylin and then observed under a light microscope (Olympus BH2, Japan).

Immunohistochemistry scoring. The immunohistochemistry scoring results for cyclin D1 were based on staining intensity and frequency of distribution of immunopositive cells The tissue samples were considered positive for cyclin D1 when nuclear staining was detected in $\geq 10 \%$ of the cells. A distinct brown nuclear staining was considered as positive and cytoplasmic staining was disregarded. The intensity of nuclear staining was scored as follows: 0, no expression; $1+$, mild expression; $2+$, moderate expression; and $3+$, strong expression. The percentage of positive cells was determined after evaluating the entire lesion present in the slide. The
Table II. Cyclin D1 expression in normal, BD and SCC samples.

\begin{tabular}{lcccc}
\hline Cyclin D1 & $\begin{array}{c}\text { Control, } \\
\mathrm{n}=8(\%)\end{array}$ & $\begin{array}{c}\text { BD, } \\
\mathrm{n}=30(\%)\end{array}$ & $\begin{array}{c}\text { SCC } \\
\mathrm{n}=24(\%)\end{array}$ & P-value \\
\hline Positive & $0(0)$ & $13(43.3)$ & $17(70.8)$ & $<0.05$ \\
\hline
\end{tabular}

${ }^{a}$ The P-value for cyclin D1 expression was $0.022,0.001$ and 0.043 between the control and BD groups, between the control and SCC groups and between the BD and SCC groups, respectively. All the differences were considered to be statistically significant $(\mathrm{P}<0.05)$. $\mathrm{BD}$, Bowen's disease; SCC, squamous cell carcinoma.

Table III. Cyclin D1 expression and squamous cell carcinoma grading.

\begin{tabular}{lccc}
\hline & \multicolumn{3}{c}{ Cyclin D1 expression } \\
\cline { 2 - 4 } Grade & Positive, $\mathrm{n}$ & Negative, $\mathrm{n}$ & P-value \\
\hline I & 4 & 0 & $0.590^{\mathrm{a}}$ \\
I-II & 2 & 2 & \\
II & 4 & 2 & \\
II-III & 6 & 2 & \\
III & 1 & 1 &
\end{tabular}

${ }^{a}$ Not statistically significant.

mean percentage of positive tumour cells for each marker was determined in 5 fields per section at high magnification and was assigned to one of the following categories: $0,<10 \%$; $1+, 11-25 \% ; 2+, 26-50 \% ; 3+, 51-75 \%$; and $4+,>75 \%$. The scores indicating the percentage of positive cells and staining intensity were multiplied to produce a semiquantitative immunohistochemical score: $0,-; 1-4$ points, $+; 5-8$ points, ++ ; and 9-12 points, +++ .

Statistical analysis. All the relevant histopathological and immunohistochemical data obtained were tabulated and subjected to appropriate statistical analysis using the SPSS 16.0 statistical software (SPSS Inc., Chicago, IL, USA). The Chi-square test was used to assess the data and $\mathrm{P}<0.05$ was considered to indicate a statistically significant difference.

\section{Results}

Cyclin D1 immunohistochemical expression. Morphologically normal skin (control) was negative for cyclin D1 immunostaining. In $\mathrm{BD}$, there was positive nuclear staining in the entire thickness of the atypical and dysplastic epithelium. In SCC, moderate to strong positive staining for cyclin D1 was observed in the nuclei of the dysplastic epithelium and the nuclei of cancer cell nests and adjacent dysplastic epidermis (Figs. 1-4).

Statistical analysis results of positive immunoexpression of cyclin DI in BD and SCC. In the present study, 13/30 (43\%) 


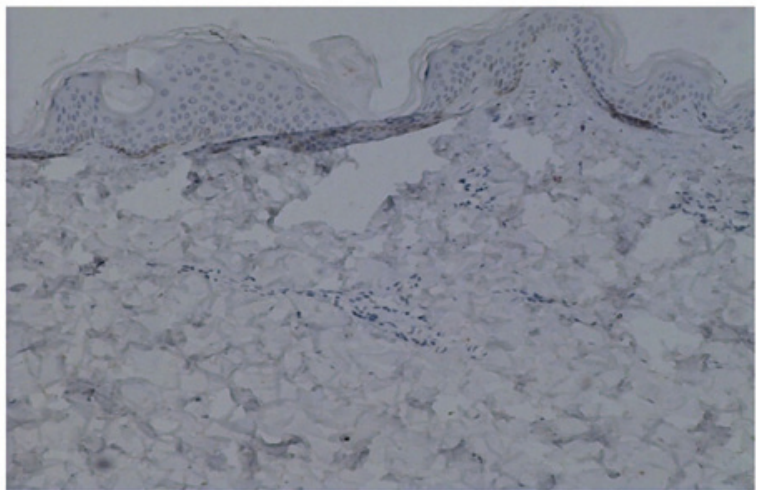

Figure 1. Immunohistochemical staining for cyclin D1 in normal skin (magnification, x100). Normal skin sections stained negative for cyclin D1.

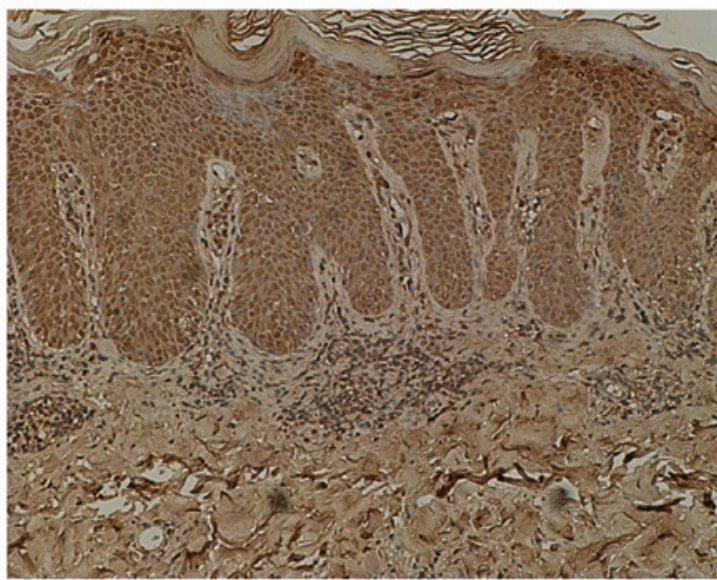

Figure 2. Immunohistochemical staining for cyclin D1 in Bowen's disease (magnification, x100). Strong positive staining for cyclin D1 was present in the entire thickness of the atypical and dysplastic epithelium in Bowen's disease.

of the BD cases and 17/24 (70.8\%) of the SCC cases exhibited positive expression for cyclin D1. Cyclin D1 was undetectable in all 8 normal skin samples. The difference between the control and cancer groups was statistically significant $(\mathrm{P}<0.05)$. The expression of cyclin D1 in SCC was significantly higher compared to that in the $\mathrm{BD}$ group $(\mathrm{P}<0.05)$ (Table II). However, there was no association between the expression of cyclin D1 and SCC grading (Table III).

\section{Discussion}

The cyclin D1 protein belongs to the highly conserved cyclin family, whose members are characterized by a significant periodicity in protein abundance throughout the cell cycle. Altered expression of the cell-cycle regulatory genes involved in the cyclin D1/p16 INK4-pRb pathway may contribute to epidermal hyperproliferation (18). Cyclin D1 is a member of the G1 cyclin family that regulates the transition through the G1 phase of the cell cycle. The CCND1 gene is located on chromosome $11 \mathrm{q} 13$ and exhibits the characteristics of a cellular oncogene. Genetic aberrations in the regulatory circuits that control the transition through the G1 phase of the cell cycle are frequently encountered in human cancer and the overexpression of cyclin D1 is one of the most commonly observed abberations.

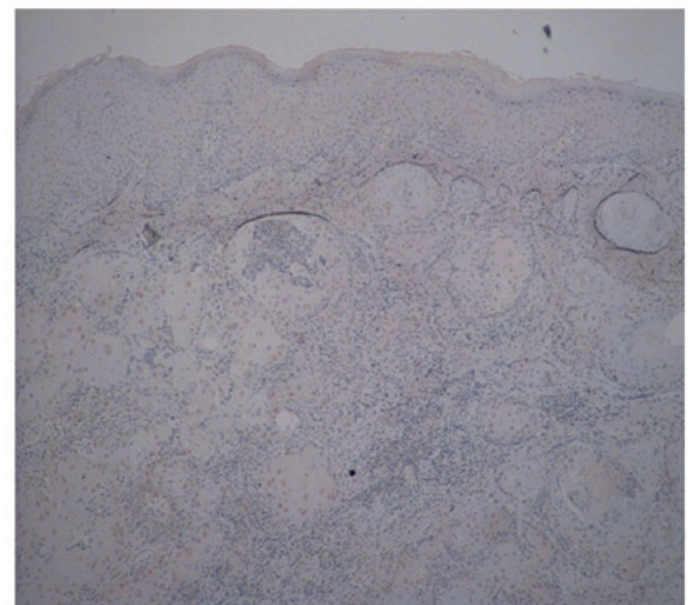

Figure 3. Immunohistochemical staining for cyclin D1 in squamous cell carcinoma (SCC) (magnification, x40). Moderate staining for cyclin D1 was present in the nuclei of the dysplastic epidermis and the cancer cell nests in SCC.

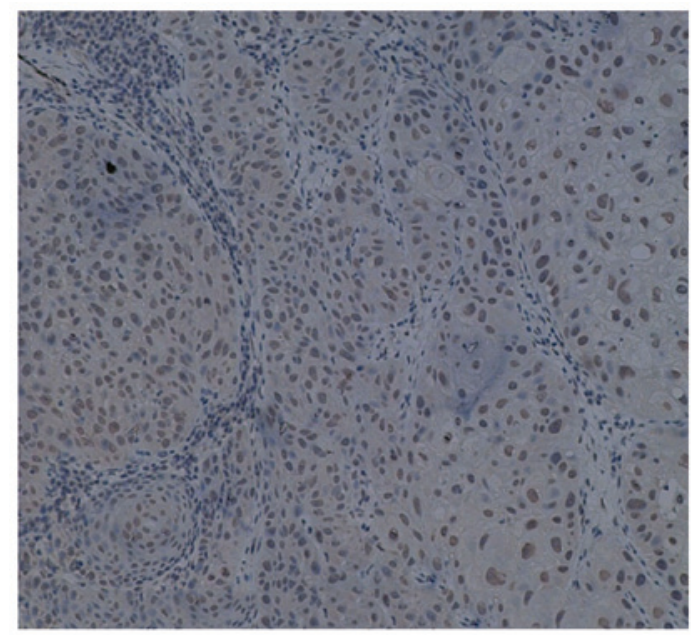

Figure 4. Immunohistochemical staining for cyclin D1 in squamous cell carcinoma (SCC) (magnification, x100). Positive nuclear staining for cyclin D1 was present in the cancer cell nests in SCC.

The role of cyclin D1 has been extensively investigated in the cell nucleus as a regulatory subunit of the cyclin-dependent kinase $\mathrm{Cdk} 4 / 6$, which is involved in the regulation of proliferation and development in mammals (19). The overexpression of cyclin D1 may predispose to skin carcinogenesis, whereas loss of cyclin D1 reduces tumor formation. The mechanisms underlying cyclin D1 overexpression in cancer include gene amplification, chromosomal translocation and mitogenic stimulation of gene transcription. Overexpression of cyclin D1 has been reported in several different types of human cancers (20) and is generally associated with poor prognosis of SCC at different sites $(21,22)$. Alterations of CCND1 are among the most common genetic changes in cancer $(23,24)$, while cyclin D1 overexpression is associated with elevated cell proliferation. In the present study, it was observed that the positive rate of cyclin D1 expression in BD was $43.3 \%(13 / 30)$, whereas the positive rate in SCC was $70.8 \%$ (17/24). There was a statistically significant difference between the control and the two cancer groups $(\mathrm{P}<0.05)$. Additionally, the expression of 
cyclin $\mathrm{D} 1$ in SCC was higher compared to that in $\mathrm{BD}$ and the control groups $(\mathrm{P}<0.05)$. Huang et al $(25)$ reported a marked overexpression of cyclin D1 in cutaneous SCC compared to that in normal skin, which is in line with the data reported for several other malignancies, including head and neck SCC (3) and oropharyngeal SCC (16), including a large number of samples. The prognostic value of cyclin D1 has been described in detail for tumor entities in these experiments.

However, whether cyclin D1 expression correlates with histopathological differentiation in tumors remains controversial. Saawarn et al (16) observed that there was an increase in cyclin D1 expression with increasing histological differentiation in oral SCC. However, Liang et al (14) reported that the expression of cyclin D1 was markedly increased from normal skin through dysplasia to $\mathrm{BD}$, but was not significantly correlated with the degree of SCC differentiation. The results demonstrated that the effect of cyclin D1 overexpression is restricted to proliferation of cells, conferring a growth advantage, but does not promote their differentiation (14). In the present study, no statistically significant difference $(P>0.05)$ was identified in the expression of cyclin D1 among different degrees of SCC differentiation (4 grade I, 4 grade I-II, 6 grade II, 8 grade II-III and 2 grade III cases). These observations indicated that the expression of cyclin D1 increases from normal skin to cancer, but it is not associated with the degree of SCC differentiation, suggesting that, during the process of skin tumorigenesis, the overexpression of cyclin D1 mainly affects the proliferation of tumour cells rather than their differentiation.

In conclusion, the abovementioned findings suggest that, although the amplification of cyclin D1 is not correlated with the degree of SCC differentiation, the overexpression of cyclin D1 plays a significant role in neoplastic transformation and may be a significant marker during the early phases of multistage skin carcinogenesis.

\section{Acknowledgements}

This study was supported by Huashan Hospital and Fudan University.

\section{References}

1. Calzado MA, Renner F, Roscic A, et al: HIPK2: a versatile switchboard regulating the transcription machinery and cell death. Cell Cycle 6: 139-143, 2007.

2. Grimm M, Krimmel M, Alexander D, et al: Prognostic value of histamine $\mathrm{H} 1$ receptor expression in oral squamous cell carcinoma. Clin Oral Investig 17: 949-955, 2013.

3. Hanken H, Gröbe A, Cachovan G, et al: CCND1 amplification and cyclin D1 immunohistochemical expression in head and neck squamous cell carcinomas. Clin Oral Investig 18: 269-276, 2014

4. Angadi PV and Krishnapillai R: Cyclin D1 expression in oral squamous cell carcinoma and verrucous carcinoma: correlation with histological differentiation. Oral Surg Oral Med Oral Pathol Oral Radiol Endod 103: e30-e35, 2007.

5. Fracchiolla NS, Pruneri G, Pignataro L, et al: Molecular and immunohistochemical analysis of the bcl-1/cyclin D1 gene in laryngeal squamous cell carcinomas: correlation of protein expression with lymph node metastases and advanced clinical stage. Cancer 79: 1114-1121, 1997.
6. Bedewy AM, Mostafa MH, Saad AA, et al: Association of cyclin D1 A870G polymorphism with two malignancies: acute lymphoblastic leukemia and breast cancer. J BUON 18: 227-238, 2013.

7. Shi XY, Bhagwandeen B and Leong AS: p16, cyclin D1, Ki-67, and AMACR as markers for dysplasia in Barrett esophagus. Appl Immunohistochem Mol Morphol 16: 447-452, 2008.

8. Parekh P, Motiwale L, Naik N, et al: Downregulation of cyclin D1 is associated with decreased levels of p38 MAP kinases, Akt/PKB and Pak1 during chemopreventive effects of resveratrol in liver cancer cells. Exp Toxicol Pathol 63: 167-173, 2011.

9. Chen L, Wei T, Si X, et al: Lysine acetyltransferase GCN5 potentiates the growth of non-small cell lung cancer via promotion of E2F1, cyclin D1, and cyclin E1 expression. J Biol Chem 288: 14510-14521, 2013.

10. Chrysovergis A, Gorgoulis VG, Giotakis I, et al: Simultaneous over activation of EGFR, telomerase (h TERT), and cyclin D1 correlates with advanced disease in larynx squamous cell carcinoma: a tissue microarray analysis. Med Oncol 28: 871-877, 2011.

11. Fristrup N, Birkenkamp-Demtröder K, Reinert T, et al: Multicenter validation of cyclin D1, MCM7, TRIM29, and UBE2C as prognostic protein markers in non-muscle-invasive bladder cancer. Am J Pathol 182: 339-349, 2013.

12. Bae DS, Cho SB, Kim YJ, et al: Aberrant expression of cyclin D1 is associated with poor prognosis in early stage cervical cancer of the uterus. Gynecol Oncol 81: 341-347, 2001.

13. Cao W, Liu Y, Zhang H, Wang S, et al: Expression of LMP-1 and cyclin D1 protein is correlated with an unfavorable prognosis in nasal type NK/T cell lymphoma. Mol Med Rep 1: 363-368, 2008.

14. Liang SB, Furihata M, Takeuchi T, et al: Overexpression of cyclin D1 in nonmelanocytic skin cancer. Virchows Arch 436: 370-376, 2000.

15. Saida T, Koga $\mathrm{H}$ and Uhara H: Key points in dermoscopic differentiation between early acral melanoma and acral nevus. J Dermatol 38: 25-34, 2011.

16. Saawarn S, Astekar M, Saawarn N, et al: Cyclin D1 expression and its correlation with histopathological differentiation in oral squamous cell carcinoma. ScientificWorldJournal 2012: 978327, 2012.

17. Dicker T, Siller G and Saunders N: Molecular and cellular biology of basal cell carcinoma. Australas J Dermatol 43: 241-246, 2002.

18. Lu X, Du J, Liang J, et al: Transcriptional regulatory network for psoriasis. J Dermatol 40: 48-53, 2013.

19. Fernández-Hernández R, Rafel M, Fusté NP, et al: Cyclin D1 localizes in the cytoplasm of keratinocytes during skin differentiation and regulates cell-matrix adhesion. Cell Cycle 12: 2510-2517, 2013.

20. Todd R, Hinds PW, Munger K, et al: Cell cycle dysregulation in oral cancer. Crit Rev Oral Biol Med 13: 51-61, 2002.

21. Müller W, Noguchi T, Wirtz HC, et al: Expression of cell-cycle regulatory proteins cyclin $\mathrm{D} 1$, cyclin $\mathrm{E}$, and their inhibitor $\mathrm{p} 21$ WAF1/CIP1 in gastric cancer. J Pathol 189: 186-193, 1999.

22. Rodriguez-Puebla ML, LaCava M and Conti CJ: Cyclin D1 overexpression in mouse epidermis increases cyclin-dependent kinase activity and cell proliferation in vivo but does not affect skin tumor development. Cell Growth Differ 10: 467-472, 1999.

23. Elsheikh S, Green AR, Aleskandarany MA, et al: CCND1 amplification and cyclin D1 expression in breast cancer and their relation with proteomic subgroups and patient outcome. Breast Cancer Res Treat 109: 325-335, 2008.

24. Holst F, Stahl PR, Ruiz C, Hellwinkel O, et al: Estrogen receptor alpha (ESR1) gene amplification is frequent in breast cancer. Nat Genet 39: 655-660, 2007.

25. Huang K, Huan C, Shan K, et al: Significance of PC cell-derived growth factor and cyclin D1 expression in cutaneous squamous cell carcinoma. Clin Exp Dermatol 37: 411-417, 2012.

26. Scantlebury JB, Luo J, Thorstad WL, et al: Cyclin D1-a prognostic marker in oropharyngeal squamous cell carcinoma that is tightly associated with high-risk human papillomavirus status. Hum Pathol 44: 1672-1680, 2013. 